REVISTA

dela

$C E P A L$

NUMERO 55

AERIL 1995

BANTIAGO DE CHILE

ANIBAL PINTO

Director

EUGENIO LAHERA

Secretario Técnico

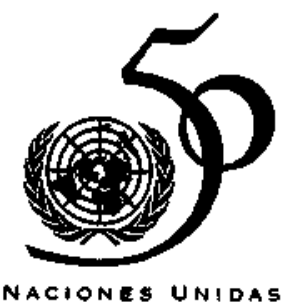


Una síntesis de la propuesta de la CEPAL

Eugenio Lahera, Ernesto Ottone y Osvaldo Rosales

Consolidación de la paz después de los conflictos:

un desafío para las Naciones Unidas

Graciana del Castillo

Descentralización y democracia: el nuevo municipio latinoamericano

Eduardo Palma

Economía política del proteccionismo después de la Ronda Uruguay

Política comercial e inserción internacional.

Una perspectiva latinoamericana

Marta Bekerman y Pablo Sirlin

Movimientos de capital y financlamiento externo

Benjamín Hopenhayn

Impacto de la política camblaria y comercial sobre el desempeño

exportador en los años ochenta

Graciela Moguillansky

Situación y perspectivas ambientales en América Latina y el Caribe

Nicolo Gligo

Expectativas de la juventud y el desarrollo rural

Martine Dirven

Empresas transnacionales y cambios estructurales en la industria de Argentina, Brasil, Chile y México

Ricardo Bielschowsky y Giovanni Stumpo

El Salvador: política industrial, comportamiento empresarial y perspectivas

Roberto Salazar

El cambio tecnológico en los análisis estructuralistas

Armando Kuri

Orientaciones para los colaboradores de la Revista de la CEPAL 


\section{Política comercial \\ e inserción internacional. Una perspectiva \\ latinoamericana}

\section{Marta Bekerman Pablo Sirtln}

Investigadores del Centro de Estudios de la Estructura EConómica (CENES),

Universidad de Buenos Aires.
En este artículo se examinan lineamientos de la política comercial que deberá utilizar la región para lograr una inserción internacional dinámica, tomando en cuenta el contexto internacional, las principales derivaciones normativas de los debates téricos sobre el tema y algunas lecciones que se pueden extraer de los casos exitosos. Se plantea que en los países de la región la política comercial puede ser instrumento de gestión macroeconómica, de gestión fiscal y, a nivel microeconómico, de asignación de recursos; su utilización con diversos objetivos como instrumento suboptimo se justifica cuando los óptimos se hallan restringidos (por ejemplo, en la política cambiaria). Se sostiene además que es necesaria una estrecha coordinación de las políticas comerciales, industriales y tecnológicas, para asegurar altos niveles de inversión en los sectores transables de la economía, una competitividad basada en crecientes incrementos de la productividad y una mejora en el perfil de especialización de la región. Por último, se destaca la importancia de fortalecer la institucionalidad en los Estados latinoamericanos para garantizar a sus intervenciones un nivel adecuado de eficacia. 


\section{Introducción}

La consolidación de un proceso de crecimiento de lar. go plazo en los países de América Latina está estrechamente vinculada a una inserción internacional dinámica. De allí la importancia de definir una política comercial a la altura de los desafíos que enfrenta la región. Ahora bien, las publicaciones especializadas indican que el conocimiento y el avance científico técnico son factores que determinan el desarrollo de nuevas ventajas comparativas. Esto vuelve cada vez mas oscuros los límites entre la política comercial, la industrial y la tecnológica. En otras palabras, hablar de política comercial en sentido restringido (aranceles, barreras no arancelarias, reintegros a la exportación, etc.) puede darnos una idea sólo incompleta de la estrategia de reestructuración que lleva adelante un país.

Este artículo se propone entonces analizar, tomando en cuenta diversos aportes de los escritos te6ricos y de la experiencia internacional, el rol de la política comercial en los campos en que la intervención pública puede contribuir a mejorar la inserción internacional de América Latina. Para ello se examinará aquí el contexto internacional y las perspectivas planteadas por la recientemente finalizada Ronda Uruguay del GATT (sección II). ${ }^{\mathrm{I}}$ Se evaluará la dimensión de la política comercial, como instrumento de gestión macroeconómica (sección III) y en el marco de las políticas microeconómicas horizontales y específicas (sección IV). Se hará referencia a las restricciones que impone la limitada capacidad de las instituciones públicas para aplicar con eficacia políticas activas (sección $\mathrm{V}$ ). Y finalmente, a la luz de los lineamientos teóricos esbozados en el trabajo y de las restricciones -internas y externas - de la realidad económica de nuestros países, se intentará formular algunas recomendaciones en materia de política comercial para los próximos años (sección VI).

\section{II}

\section{El contexto internacional y}

\section{las perspectivas del GATT}

En los años ochenta se profundizaron en la economía internacional los procesos de globalización y de regionalización.

La globalización económica, entendida como una creciente interdependencia de las distintas naciones, se manifestó en que las tasa de expansión del comercio internacional y fundamentalmente de la inversión extranjera directa (IED) y de los flujos financieros, superaron ampliamente la del producto bruto mundial. ${ }^{2}$

El fuerte aumento del comercio mundial se remonta al período de posguerra, cuando se inició un proceso de liberalización a través de sucesivas rebajas en los niveles arancelarios en el marco de las rondas del GATT. El crecimiento de la IED fue estimulado, a su vez, por el intento de contrarrestar potenciales medidas proteccionistas y la necesidad de establecer bases competitivas más fuertes (tanto en lo que hace a la comercialización como a la incorpora- ción tecnológica). Por último, el aumento de la globalización financiera fue potenciado por los crecientes desequilibrios comerciales, por los avances tecnológicos en el campo de la información y las comunicaciones, y por el proceso de desregulación de las operaciones financieras a nivel mundial.

En este contexto, signado a su vez por la aceleración de la innovación tecnológica, existe consenso en que una estrategia exportadora favorecerá más la absorcion y adaptación de tecnología y, por ende, el

\footnotetext{
1 En el anexo de este trabajo se presenta una descripción de los nuevos instrumentos incorporados recientemente por la Ronda Uruguay para aumentar la disciplina en el manejo de las políticas comerciales.

2 En efecto, entre 1983 y 1989 las inversiones directas crecieron a una tasa tres veces mayor que la de incremento de las exportaciones mundiales y cuatro veces superior a las de crecimiento del PIB mundial (Guerra Borges, 1993)
} 
desarrollo económico, que un régimen orientado exclusivamente al mercado interno.

Así, la creciente interdependencia económica plantea la conveniencia de una mayor inserción de las economías en el mercado internacional, al mismo tiempo que obliga a redefinir los grados de libertad de que disponen las políticas nacionales.

Cabe señalar aquí dos aspectos que caracterizan este proceso de globalización: el carácter asimétrico de su relación con los países periféricos, y las contradicciones que presenta.

Ese carácter asimétrico se manifiesta en el hecho de que dos tercios del comercio mundial se concentran actualmente en Estados Unidos, la CEE y Japón, países que en la segunda mitad de los años ochenta recibieron más del $80 \%$ de la IED, mientras los países en desarrollo recibían solamente el $17 \%$ del total, y dos terceras partes de este monto se dirigían a sólo 10 naciones.

Por su parte, las contradicciones del proceso de globalización se manifiestan en un considerable aumento de presiones proteccionistas de nuevo tipo ejercidas por los países industrializados. ${ }^{3}$ En efecto, a partir de los años setenta hubo un fuerte incremento de las barreras no arancelarias (acuerdos voluntarios de exportación, derechos compensatorios, cláusulas antidumping, etc. $)^{4}$ que al extenderse - sobre todo a sectores maduros como el de la siderurgia, los textiles y los productos agrícolas-- afectaron especialmente al comercio con los países periféricos (Laird y Nogués, 1989). ${ }^{5}$

Las causas de la irrupcion de este nuevo proteccionismo se vinculan con:

i) El fuerte aumento de las exportaciones desde Japón y las economías asiáticas de industrialización reciente hacia países industrializados, los que a partir de los años setenta exhiben bajas tasas de crecimiento. Los mayores niveles de desempleo y de capacidad ociosa consiguientes hacen que el ajuste ante la penetración de importaciones se haga más difícil (hay menos empleos nuevos para contrarrestar las pérdidas en los sectores declinantes) y que el grado de tolerancia social sea menor.

${ }^{3}$ En abierto contraste, los países en desarrollo muestran desde los años ochenta una fuerte tendencia a la apertura de sus economías.

4 Algunas estimaciones indican que hacia 1980 el $48 \%$ del comercio mundial estaba controlado (sujeto a alguna forma de barrera no arancelaría), contra un $40 \%$ en 1974 (Grimwade, 1989).

5 En los años ochenta, por ejemplo, el $44 \%$ de las acciones antidumping y el $61 \%$ de tos derechos compensatorios aplicados por Estados Unidos, la Comunidad Económica Europea, Australia y Canadá tuvieron como objetivo restringir exportaciones de países en desarrollo (Fundación U.I.A., Consejo Académico, 1994). ii) Fallas en el sistema monetario internacional que dificultan la correción — por ejemplo, a través de modificaciones de las tasas de cambio- de los fuertes desequilibrios comerciales entre países.

iii) La inexistencia de un poder hegemónico en la política comercial internacional. La autoridad y el liderazgo en materia comercial ejercido por Estados Unidos en la posguerra se fueron minando por las posiciones cada vez más proteccionistas de ese país desde mediados de los años setenta. ${ }^{6}$

Según Grimwade (1989), una razón adicional para explicar el aumento del proteccionismo reside en las fallas de las reglas y mecanismos del GATT. ${ }^{7}$ Este 6rgano - a través de la Ronda Tokio, realizada en 1979- no fue eficaz para restringir la proliferación de barreras no arancelarias. Pero, en nuestra opinión, la falta de capacidad disciplinaria del GATT en este período no fue causa del incremento de las presiones proteccionistas, sino más bien su manifestación.

Las dificultosas negociaciones de la Ronda Uruguay representaron un esfuerzo por negociar reglas globales frente a estas presiones proteccionistas. Sus resultados exhiben las mismas asimetrías señaladas más atrás. Hay un mayor disciplinamiento de los países periféricos (al limitar ciertos subsidios que ellos utilizan), así como un intento de los países industrializados de no perder grados de libertad para proteger ciertos sectores no competitivos de sus economías y para asegurar las ventajas comparativas adquiridas en los sectores tecnológicamente más avanzados (véase el anexo).

La eficacia futura del GATT dependerá de que las causas que originaron las crecientes presiones proteccionistas en el Norte puedan llegar a revertirse. Por eso, aún sus sostenedores más entusiastas señalan que su éxito, o bien está lejos de ser definitivo, o puede llegar a ser sólo parcial.

Algunos autores señalan que los acuerdos alcanzados en la Ronda Uruguay son una condición nece-

\footnotetext{
6 Motta Veiga (1988) señala que, en la búsqueda de su perdida hegemonía comercial, Estados Unidos está llevando adelante una estrategia defensiva y otra ofensiva. La primera tiene lugar en los sectores en reestructuración a través de instrumentos de protección comercial. La segunda se da en los segmentos industriales y de servicios vinculados a las nuevas tecnologias —en las que defiende el libre comercio-, busca mantener las ventajas comparativas adquiridas, y se está llevando adelante a través de negociaciones multilaterales dentro del ámbito del GATT (libre comercio de servicios, derechos de propiedad intelectual, etc.) y también de negociaciones bilaterales, a través de presiones y cláusulas de reciprocidad.

7 Especialmente en las clásusulas de salvaguardia del artículo 19 establecidas en la Ronda Tokio y que dieron lugar a que dicho artículo fuese soslayado por muchos países a través del establecimiento de barteras no arancelarias.
} 
saria, pero no suficiente, para que el sistema mundial alcanze la "integración profunda" requerida por el proceso de globalización. ${ }^{8}$ Es más, sostienen que, en una primera etapa, esa integración sólo tendrá lugar a nivel de los acuerdos comerciales regionales, que están llamados a tener un crecimiento inevitable por lo menos durante los años noventa (Lawrence, 1993). ${ }^{9}$

La regionalización de los mercados - la otra tendencia importante de la economía internacional-, es un factor más que podría poner trabas al éxito del GATT y favorecer el aumento de las asimetrías ya existentes. Para los países incluidos en los principales bloques comerciales existentes o en formación (Unión Europea, NAFTA, Asia-Pacífico), la integración económica asegura una mayor competencia, una mayor escala productiva y un mayor acceso a los mercados. Por el contrario, los países excluidos pueden quedar más expuestos a posibles conflictos comerciales, o al desvío del comercio y de la IED hacia países pertenecientes a dichos bloques.
En otras palabras, el acceso a los mercados será más seguro para los países que tienen la oportunidad de unirse a bloques regionales. Aun las empresas menos eficientes de los países que se integran pueden llegar a amagar los mercados de exportación de productores más eficientes en virtud de las mayores economías de escala que la ampliación del mercado permite (Hughes Hallets y Primo Braga, 1994).

Las asimetrías que presenta el proceso de globalización, en un clima de incertidumbre sobre la efectividad que alcanzará el GATT y de fuerte avance de los procesos de regionalización, ponen de relieve la importancia de profundizar los vínculos regionales entre los países latinoamericanos del Cono Sur. ${ }^{10}$ Además de los beneficios económicos potenciales que pueden derivar de la ampliación de los mercados, la consolidación de estos espacios regionales puede contribuir a aumentar la capacidad de negociación del área frente a otros países y bloques comerciales, así como también dentro del propio GATT.

III

\section{La política macroeconómica, la inversión} en sectores transables y la política comercial

La primera condición necesaria para lograr la inserción internacional adecuada es disponer de niveles apropiados de inversión en los sectores en que la economía se especializará internacionalmente. Esto abre a la política macroeconómica campos de acción que van desde los incentivos a la inversión en general, hasta los incentivos a la inversión en los sectores transables, y en los no transables.

Para lograr un ambiente favorable a la inversión es fundamental, ante todo, asegurar un marco global de estabilidad que permita planificar a más largo plazo y lograr que el sistema de precios relativos ofrezca

\footnotetext{
8 Según Lawrence (1993) la integración profunda implica avanzar más allá de la eliminación de barreras fronterizas hacia la compatibitización de aquellas políticas que pueden discriminar, en forma inadvertida, en contra de otros países.

9 Hughes Hallets y Primo Braga (1994) señalan que es más fácil cumplir con objetivos de coordinación de políticas a nivel regional que a nivel multilateral, porque en este último caso es difícil demostrar un compromiso creíble de todos los participantes.
}

la información y los alicientes necesarios para aprovechar mejor las ventajas comparativas." Igualmente importantes son las políticas públicas que influyen en los parámetros de las funciones de ahorro e inversión, así como una organización eficiente del sistema financiero que permita canalizar los ahorros hacia proyectos productivos. ${ }^{12}$ En su dimensión macroeconómica, la política comercial puede desempeñar por lo menos dos papeles importantes: como instrumento ge-

\footnotetext{
10 Wonacott y Wonaccott (1981) demuestran teóricamente la superioridad de la integración regional sobre la apertura comercial en aquellos casos en que los países enfrentan barteras proteccionistas en los mercados mundiales.

II La estabilidad macroeconómica es además una condición previa necesaria para asegurar la efectividad de las políticas microeconómicas (Rodrik, 1993).

${ }^{12}$ Estos aspectos, y fundamentalmente la existencia de mecanismos de financiamiento de corto y largo płazo a tasas adecuadas, parecen constituir un factor indispensable para consolidar un aparato de producción genuinamente competitivo. Por ser éste un tema muy conocido, no será tratado aquí con mayor amplitud.
} 
nerador y distribuidor de recursos fiscales, y como uno de los elementos determinantes del tipo de cambio efectivo real.

El primero cobra relevancia porque la consolidación del frente fiscal parece constituir una condición imprescindible para la estabilidad macroeconómica. De la teoría neoclásica de las distorsiones intemas se desprende que, al no contarse con impuestos no "distorsivos" (el mítico impuesto de suma fija) y al existir importantes costos y limitaciones en la capacidad de recaudación fiscal, la política comercial puede entrar en escena como instrumento fiscal subóptimo (Corden, 1974). ${ }^{13} \mathrm{El}$ grado en que conviene recurrir a la política comercial como instrumento de recaudación debe ser, sin embargo, sopesado con los posibles costos distorsivos derivados del manejo de la política arancelaria. Por otro lado, el aumento de la recaudación puede quedar neutralizado si, para evitar el sesgo antiexportador, se incrementan al mismo tiempo los incentivos fiscales a la exportación.

El segundo aspecto resulta fundamental ya que, además de un marco macroeconómico sólido que aliente la inversión en general, es imperativo lograr que una fracción suficiente de ella se dirija al sector de bienes transables. El precio clave que define los incentivos para invertir en sectores transables o no transables es el tipo de cambio efectivo real (de exportación o de importación). Surge aquí un problema: a diferencia de lo que supone implícitamente la teoría de las ventajas comparativas, el tipo de cambio real no se ajusta automáticamente a su nivel de equilibrio (al menos, a aquel que asegura el equilibrio de la balanza comercial en un marco de pleno empleo).

Tanto los escritos teóricos como la experiencia de los países asiáticos destacan la importancia de un tipo de cambio real alto y estable (con la misma justificación teórica que la estabilidad macroeconómica en general). En un proceso de apertura comercial como el que viven numerosos países de la región, el tipo de cambio real que equilibra la balanza comercial puede llegar a ser más alto por la necesidad de incentivar una reasignación de recursos hacia el sector de bienes transables (CEPAL, 1990; Fritsch y Franco, 1992). ${ }^{14}$ Las experiencias exitosas de apertura exportadora (como la de la República de Corea) ilustran la aplicación de políticas que combinaron simultáneamente la apertura comercial con la devaluación del tipo de cambio real (Amsden, 1986).

Cuando en medio de procesos de estabilización se pierde la capacidad de fijar el tipo de cambio real, ${ }^{15}$ los procesos de ajuste externo pueden tornarse sumamente costosos, dado que el nivel de actividad se convierte en la principal variable de ajuste. En este contexto, la política comercial, actuando como instrumento de naturaleza puramente macroeconómica, puede ser utilizada para compensar posibles desvíos del tipo de cambio real. ${ }^{16}$

\section{IV}

\section{Las políticas microeconómicas}

\section{y la política comercial}

A medida que se consolida el proceso de estabilización macroeconómica, empieza a manifestarse la necesidad de llevar a cabo una serie de políticas adicionales, de carácter netamente diferente de las de ajuste. Estas políticas llevan distintos rótulos: microeconómicas, mesoeconómicas, de competitividad sistémica y otros. Por razones de simplicidad, en este trabajo llamaremos políticas microeconómicas (horizontales o específicas) a todas aquellas que por su naturaleza y objetivos apuntan fundamentalmente a mejorar la

${ }^{13} \mathrm{Si}$ se rastrean, por ejemplo, las frecuentes modificaciones de la estructura arancelaria argentina desde fines de los años ochenta, se verá que en muchos casos los considerandos de las resotuciones que elevaban el nivel de protección tenían un objetivo fiscal. productividad y el patrón de especialización internacional de la economía.

\section{Las polítlcas horizontales}

a) La remoción del sesgo antiexportador

La existencia de sectores de bienes transables

\footnotetext{
14 Ello es así porque las mejoras de eficiencia derivadas no suelen ser, al menos en el corto plazo, tan importantes como para compensar el efecto negativo inicial sobre el balance comercial.

is Ya sea por la utilización de un ancla cambiaria en un contexio de rigidez a la baja de los precios nominales (ausencia de deflación), por una modalidad dolarizada de formación de precios, 0 por el impacto de la afluencia de capitales desde el exterior.

16 En el mismo sentido actúan todas aquellas políticas (comerciales o de otro tipo) que intentan reducir los costos del sector expontador.
} 
con niveles de productividad adecuados no garantiza que éstos se destinen al mercado internacional. En efecto, además de la rentabilidad absoluta de las operaciones de exportación (que se determina básicamente por la eficiencia comparativa de los distintos sectores y el tipo de cambio), se debe tomar en cuenta la rentabilidad relativa de ellas en relación con las ventas al mercado interno. En este aspecto la política comercial desempeña un papel decisivo.

Como señalamos en la sección anterior, la política comercial puede ser usada -en determinados contextos- para compensar posibles desviaciones del tipo de cambio real. Pero para cumplir con este objetivo sin generar nuevas distorsiones, es necesario que las barreras a las importaciones y los incentivos a las exportaciones se muevan en forma simultánea y proporcional. Por el contrario, las barreras arancelarias no compensadas con estímulos a la exportación desembocan en un desincentivo general al comercio conocido como sesgo antiexportador o sesgo anticomercio. ${ }^{17}$

El principal argumento para desarrollar políticas con sesgo antiexportador (el del arancel óptimo por el cual una nación grande puede utilizar su poder monopólico o monopsónico para mejorar los términos de su intercambio) es difícilmente aplicable a los países de la región (salvo casos aislados, respecto de ciertos recursos naturales escasos).

Las experiencias del Sudeste asiático (especialmente la sudcoreana y taiwanesa) demuestran, sin embargo, que para evitar el sesgo antiexportador no es necesario un régimen de total apertura comercial. El mismo resultado se puede lograr a través de mecanismos administrativos (regímenes de reintegros, admisión temporaria, zonas de procesamiento de exportaciones, etc.) que utilizados en forma eficiente brinden condiciones virtuales de libre comercio para los productores de bienes exportables (Wade, 1990).

Ahora bien, el sesgo antiexportador no sólo es inducido por las políticas comerciales. También se traduce en que el impacto de ciertas fallas de mercado sea mayor sobre las operaciones de comercio exterior que sobre las ventas internas. Entre estas fallas cabe destacar tres: la insuficiente información sobre los mercados externos, la incapacidad del mercado de capita-

\footnotetext{
${ }^{17}$ En este caso las empresas de los sectores transables evalúan como más conveniente vender en el mercado interno protegido en vez de enfrentar los menores precios existentes en el mercado internacional. A su vez, la protección comercial torna más caros los bienes importados, sesgando la demanda hacia los productos fabricados localmente. Opera entonces una reducción sirnultánea de los incentivos a exportar y a importar, que conducen a una reducción de los niveles de comercio.
}

les de proveer financiamiento para la exportación, y las economías de escala requeridas en la comercialización internacional de productos nuevos. Dado el escenario mundial descrito en la seccion II de este trabajo, las políticas públicas dirigidas a superar tales fallas adquieren cada vez más importancia si se desea ocupar nuevos nichos en los mercados externos y mejorar el perfil de especialización internacional.

Las experiencias exitosas del Sudeste asiático muestran que se han hecho importantes esfuerzos en cada una de estas áreas. El sistema de financiamiento de exportaciones que aplicó la República de Corea fue -en la opinión de algunos autores - el instrumento que más contribuyó al éxito de la estrategia exportadora de ese país (Rhee, 1989). Este sistema se canalizó a través de la banca mediante mecanismos de redescuento y de financiamiento automático establecidos por el Banco de Corea. Por otro lado, tanto coreanos como taiwaneses han enfrentado el problema de las economías de escala existentes en la comercialización internacional. La República de Corea logró estimular el desarrollo de grandes compañías comercializadoras privadas, a las que impuso exigencias específicas de capitalización, volumen de exportación y número de oficinas en el exterior. La economía taiwanesa, cuyo potencial exportador reside en la pequeña y mediana empresa, desarrolló una activa política de comercialización internacional a través de oficinas comerciales instaladas en los principales centros comerciales del mundo.

En todo caso, las recomendaciones de política que derivan de estas fallas de mercado son ampliamente conocidas y, en última instancia, todos los países las aplican en alguna medida. La diferencia estriba más bien en la intensidad con la que se aplican. $\mathrm{La}$ experiencia internacional nos muestra que han sido las economías que han ido más lejos en el desarrollo de estas políticas las que han logrado mejoras sustanciales en su inserción internacional.

b) La negociación del acceso a los mercados externos

En el enfoque neoclásico tradicional, la apertura es la mejor altemativa, aun cuando otros países apliquen políticas comerciales distorsivas (aranceles, subsidios a las exportaciones, y otras). En ese caso, lo que corresponde es recurrir a los foros internacionales para negociar la generalización de esa apertura a nivel multilateral (Krueger, 1990).

El levantamiento de algunos supuestos simplificadores (competencia perfecta en los mercados internacionales, rendimientos constantes, etc.) ha llevado 
a algunos enfoques teóricos (entre ellos la nueva teoría del comercio internacional), a diferir radicalmente de la teoría neoclásica. ${ }^{18}$ Básicamente, se pone en duda que, ante situaciones de fuerte intervencionismo extranjero, la política más conveniente sea la de libre comercio indiscriminado. Se plantea entonces la utilización de la política comercial, o simplemente la amenaza de utilizarla, como mecanismo de negociación para facilitar el acceso a mercados protegidos o subsidiados por otras naciones (Tyson, 1990; Dombusch, 1990). ${ }^{19}$ En este contexto, como se señaló en la sección II, los procesos de integración regional, como el MERCOSUR, ofrecen ventajas potenciales para fortalecer la capacidad negociadora de los países miembros frente a las asimetrías existentes en las relaciones comerciales internacionales.

\section{c) Políticas de apoyo a la productividad y política comercial}

Para consolidar un sector exportador dinámico es preciso no sólo asegurar niveles de inversión adecuados en los sectores transables, sino además mejorar permanentemente su productividad. En efecto, la competitividad puede ser mejorada en un momento dado a través de variables macroeconómicas (como el tipo de cambio) o a través de la reducción de ciertos costos (salariales directos e indirectos, tributarios, etc.) que pesan sobre el sector exportador. Sin embargo, para asegurar un proceso sostenido de crecimiento de las exportaciones que sea compatible con el mejoramiento del nivel de vida de la poblacion, es decir, para alcanzar altos niveles de competitividad genuina, se debe fomentar el incremento de la productividad (Fajnzylber, 1988).

En este campo, el papel principal lo tienen las políticas industriales y tecnologicas que apuntan, entre otras cosas, a asegurar una masa crítica de mano de obra clasificada, a desarrollar una infraestructura física y una red de proveedores adecuadas y a impulsar la investigación y el desarrollo tecnológico.

En el mismo sentido actúan las políticas que se orientan a compensar fallas de información en los mercados, como las de extensión industrial, asesoramiento a la pequeña y mediana empresa, mejoras en la coordinación de los agentes productivos y otras.

\footnotetext{
IR Véase, por ejemplo, una compilación de trabajos sobre política comercial estratégica en Krugman (1987).

19 Este enfoque ha influido mucho en la actual política comercial de Estados Unidos. Ya nos hemos referido a las negociaciones bilaterales - a través de cláusulas de reciprocidad- que lleva adelante este país en segmentos vinculados con las nuevas tecnologías.
}

Algunos autores sostienen la importancia de que, como paso previo a la incorporación de bienes de capital, las empresas realicen cambios en las tecnologías de organización productiva que respondan al nuevo paradigma tecnológico, con la introducción de sistemas de calidad total, de producción justo a tiempo y otros (Kaplinsky, 1988). De allí la importancia de que el Estado, a través de instituciones especializadas, ayude al sector privado (fundamentalmente a la pequeña y mediana empresa) en ese proceso de reestructuración.

Los instrumentos de política comercial pueden cumplir en este campo un papel indirecto, aunque importante. En primer lugar, las políticas dirigidas a abaratar la compra de bienes de capital (mediante la reducción o eliminación de los aranceles ${ }^{20}$ indudablemente favorecen la modernización del parque industrial. Sin embargo, al fijar los aranceles de los bienes de capital debe tenerse en cuenta no solamente su incidencia en los costos industriales, sino también los costos de oportunidad de los recursos fiscales comprometidos y los posibles efectos sobre los productores locales de bienes de capital, ya que este sector puede ser un importante transmisor del progreso tecnológico.

En segundo lugar, cabe mencionar regímenes que fomenten la especialización productiva, como el Régimen de Especialización Industrial argentino que lleva en muchos casos a generar un sesgo proexportador. En el marco de un análisis estático, este sesgo es tan disfuncional como el antiexportador. Sin embargo, en las etapas iniciales de un proceso de reestructuración los incentivos altos a la especialización pueden ser una herramienta efectiva para vencer la inercia derivada de los costos de reconversión.

En tercer lugar, se puede señalar que muchos parses han utilizado exitosamente el comportamiento exportador como mecanismo para evaluar el otorgamiento de distintos tipos de incentivos a la inversión y al desarrollo tecnologico. Este expediente -que confiere a tales instrumentos una dimensión de política comercial- tiene su fundamento racional en que el efectivo acceso al mercado internacional en ciertos sectores productivos es prueba bastante fidedigna de la capacidad desarrollada por esos sectores para producir bienes con niveles aceptables de precio y calidad. Aunque estos instrumentos no son necesariamente óptimos desde el

\footnotetext{
${ }^{20}$ Esta política puede ser vista como un instrumento microeconómico que apunta a elevar la productividad de la economía o, en forma más general, como un método indirecto para elevar el tipo de cambio real.
} 
punto de vista de la teoría económica, pueden constituir mecanismos suboptimos cuando la capacidad pública de control o la complejidad tecnológica de los bienes hace demasiado engorrosa la tarea de verificar el cumplimiento de metas de precio y calidad. De todas maneras, la posibilidad de utilizar de esta manera la política comercial estará crecientemente acotada por las nuevas normas del GATT (véase el anexo).

Por último, la formación de bloques comerciales regionales constituye una política de naturaleza comercial que puede tener fuerte impacto en la eficiencia productiva. Al someter a los productores locales a una mayor competencia, y a la vez brindarles acceso preferencial a un mercado mayor, puede darse un fuerte impulso al proceso de reconversión y a las economías de escala y de especialización. Asimismo, el comercio entre países similares puede dar pie a una mayor calificación de los trabajadores (Amsden, 1986) y de la actividad innovativa (Rodrik, 1993).

\section{Las políticas especfilicas y la política comercial}

Hasta aquí se han analizado instrumentos de política macroeconómica o microeconómica que afectan la competitividad general, sin circunscribirse, al menos explícitamente, a determinados tipos de industrias o de actividad. Ahora abordaremos un problema diferente, el de dilucidar si la autoridad económica debe permanecer neutral respecto del tipo de sectores en los que se especialice la nación o si, por el contrario, existen fundamentos para incentivar ciertos sectores.

En la versión más simplificada de la teoría neoclásica, todos los sectores son considerados equivalentes. En este marco, la política optima es la de libre comercio, que permite aprovechar al máximo las ganancias provenientes de la especialización internacional. A la inversa, las políticas selectivas distorsionan el patrón de ventajas comparativas y reducen el bienestar.

Sin embargo, de análisis más sofisticados surgen diversos argumentos teóricos en favor de la formulación de políticas selectivas, incluso a nivel de la política comercial.

Dentro de la teoría neoclásica, la existencia de fallas de mercado puede dar lugar a argumentos en favor de la formulación de políticas comerciales de carácter selectivo. ${ }^{2 !}$ Dado que numerosas fallas de

21 Mucho se ha escrito sobre la teoría de las distorsiones internas analizada aquí. Véase un texto esclarecedor en Corden (1974), y una síntesis analítica en Martirena-Mantel (1988). mercado (externalidades, indivisibilidades, regulaciones o impuestos distorsivos, etc.) afectan de manera diferente a las distintas actividades productivas, el patrón de especialización internacional que resulte de una política de libre comercio podría no ser óptimo. En la mayoría de los casos, las distorsiones del mercado son de carácter interno y las políticas optimas para corregirlas no pasan por la utilización de la política comercial. Sin embargo, esta política puede asumir - ante la falta de instrumentos optimos- - un papel subóptimo..$^{22}$ Mientras que las políticas óptimas pueden ser formalmente neutrales (por ejemplo, las medidas para superar fallas en los mercados de capitales), la política comercial subóptima probablemente deba asumir un carácter selectivo (protección más alta a aquellos sectores que, por predominancia de empresas pequeñas y medianas, por mayor riesgo tecnológico u otras razones, son más sensibles a la falta de créditos).

De lo expuesto se puede inferir que si las políticas de apertura comercial no se acompañan de las políticas optimas destinadas a superar las fallas de mercado existentes, probablemente no conduzcan a un incremento del bienestar. Por otro lado, si existen costos en la distribución de los subsidios óptimos, la protección comercial selectiva puede llegar a constituirse en la mejor alternativa de política (Corden, 1974).

Otro argumento muy conocido y vapuleado es el de la industria incipiente. La discusión neoclásica más rigurosa limitó severamente la validez de este argumento, vinculándolo a la existencia de ciertas fallas de mercado asociadas fundamentalmente a imperfecciones en los mercados de capitales (Martirena-Mantel, 1988). ${ }^{23}$ En estos casos, una vez más, la política comercial sólo puede ser utilizada como instrumento subóptimo.

La formulación de los supuestos simplificadores de la teoría tradicional ha dado lugar a otras corrientes terricas que han retomado y potenciado la línea

\footnotetext{
${ }^{22}$ Esta utilización de la política comercial como instrumento suboptimo desempeña un papel central en la justificación teorica de aranceles escalonados (y no uniformes) según el grado de elaboración de los productos. El argumento sería que las distintas distorsiones que afectan a la producción de bienes (costos de transporte de materias primas, impuestos distorsivos, etc.) aumentan su incidencia a medida que se acumulan las etapas productivas. Al respecto se plantea que una estructura arancelaria escalonada puede legar a restablecer un esquema neutral de incentivos (Fundación U.I.A., Consejo Académico, 1994)

${ }^{23}$ A nivel empítico, las experiencias de promoción de industrias incipientes muestran niveles de eficacia muy dispares (Bell, RossLarson y Westphal, 1984).
} 
de pensamiento implícita en el argumento de la industria incipiente y a las que nos referiremos brevemente a continuación.

La nueva teoría del comercio internacional (NTCI) parte del levantamiento de los supuestos de competencia perfecta y rendimientos constantes a escala. ${ }^{24}$ Esto da lugar a una clara diferenciación entre sectores productivos en el comercio internacional, ya que algunos sectores ofrecen sólo rendimientos normales y otros ofrecen rentas monopólicas. Se postula además que la principal fuente de productividad relativa en los sectores con ingresos monopólicos no es la dotación de factores del país sino la capacidad de sus habitantes para instalar determinadas industrias y alcanzar en ellas las escalas más convenientes. El patrón de especialización incorpora de esta manera un elemento aleatorio o arbitrario (Krugman, 1988). Este puede estar vinculado a la existencia de políticas comerciales (protección arancelaria, subsidios de exportación, etc.) que favorezcan a las empresas monopólicas nacionales (Brander, 1986).

En países con mercados pequeños (sin empresas lo suficientemente grandes como para alterar las reglas de la competencia estratégica internacional), aún existe la posibilidad de aplicar políticas comerciales en aquellos sectores con rendimientos normales que utilizan economías de escala indirectas vinculadas, por ejemplo, al transporte y a la comercialización internacional (Krugman, 1988). Por otro lado, los procesos de integración regional -con el consecuente incremento en el tamaño del mercado- aumentan la posibilidad de utilizar eficazmente políticas estratégicas que permitan ganar economías de escala. Por otra parte, es necesario coordinar las políticas industriales y comerciales, ya que ellas pueden afectar la forma en que se irá cristalizando el patrón de especialización intrarregional (Ocampo, 1993).

Si bien las derivaciones pro intervencionistas han sido ampliamente criticadas por distintos autores (muchos de la misma escuela), se tiende a aceptar el hecho de que las políticas comerciales estratégicas pueden afectar el patrón de comercio internacional, lo que como ya vimos, tiene importantes connotaciones para las negociaciones internacionales. ${ }^{25}$

24 VSase, entre otros: Brander y Spencer (1981), Helpman y Krugman (1985), Brander (1986) y Grossman (1986).

${ }^{25}$ Los ejes de las críticas son: la imposibilidad de conocer a ciencia cierta las políticas más adecuadas; la posibilidad de represalias (unilaterales o provenientes de la aplicación de las normas del GATT) que pueden conducir a una situación peor que la injcial, y la
Otras corrientes terricas han puesto de relieve el papel del cambio tecnológico. En ellas destacan los autores neoschumpeterianos y los vinculados a las nuevas teorías de crecimiento económico. ${ }^{26}$ Estas corrientes (fundamentalmente la neoschumpeteriana) señalan una nueva fuente de heterogeneidad entre los sectores, al decir que el desarrollo tecnológico no es exógeno ni homogéneo entre ellos. Algunos sectores muestran mayor innovación tecnológica, lo que les permite alcanzar tasas más altas de incremento de la productividad y les da la posibilidad de apropiarse de rentas schumpeterianas en el comercio internacional. Otros asumen el papel de sectores estratégicos debido a las fuertes extemalidades que transmiten al resto de la estructura productiva mediante la difusión de las innovaciones tecnologicas.

En cuanto a connotaciones de política, estos aportes tienen dos lecturas posibles. En primer lugar, desde el ángulo de la teoría neoclásica, de los argumentos mencionados podría desprenderse que los sectores tecnológicamente más avanzados muestran fallas de mercado más pronunciadas que el resto de la economía. $^{27}$

La segunda lectura posible rescata el hecho de que, igual que en los sectores con rendimientos crecientes, la ventaja competitiva en los sectores tecnológicamente avanzados no surge exclusivamente, de la dotación de factores, sino de los esfuerzos públicos y privados por desarrollar la capacidad de innovación tecnológica en áreas específicas. Las políticas públicas industriales y comerciales dirigidas a los sectores de vanguardia asumen pues un carácter estratégico, dado que permiten apropiarse de los beneficios extraordinarios generados por estos sectores. $^{28}$

\footnotetext{
falta de autonomía de los gobiernos para contrarrestar cabildeos corporativos (Gtossman, 1986). Se dice además que las políticas estratégicas orientadas a ciertas empresas (a fin de crear "campeones nacionales") pueden contribuir a profundizar las imperfecciones competitivas del mercado (Richardson, 1993).

26 Véase una instructiva compilación de las ideas del primer grupo de autores en Dosi, Freeman, Nelson, Silvenberg y Soete (1988). Del segundo grupo son ampliamence conocidos los trabajos precursores de Romer (1986) y Lucas (1988).

${ }^{27}$ Ejemplos de este tipo de fallas son, entre otros, las vinculadas con la creación de capital humano (que es utilizado con intensidad diferente por los distintos sectores) y las relacionadas con el mercado de capitales (sesgada en contra de proyectos con riesgo tecnológico y largo tiempo de maduración).

${ }^{28}$ En efecto, los mismos autores de la nueva teoría del comercio internacional fueron desplazando su interés desde las economías de escala estáticas hacia las economías de aprendizaje e innovación tecnologicos. Véase, por ejemplo, Grossman y Helpman (1991).
} 


\section{Las limitaciones institucionales}

Aunque los análisis teóricos comentados antes conducen a justificar la utilización de políticas comerciales e industriales activas, no existe en la práctica un consenso unánime sobre su conveniencia. Una serie de argumentos contrarios apunta básicamente a poner en duda la capacidad de las oficinas públicas para diseñar, aplicar y controlar con eficacia las políticas teóricamente optimas (especialmente las de carácter selectivo). Tres son los tipos de problemas que se señalan generalmente: la incapacidad del sector público para obtener toda la información necesaria, las ineficiencias propias de los aparatos administrativos públicos y la generación de conductas perversas en el sector privado ligadas a la búsqueda improductiva de ingresos. ${ }^{29}$

Si bien no es objeto de este trabajo analizar en profundidad tales problemas, hay algunos elementos de juicio que deben tenerse en cuenta al evaluar la conveniencia de llevar a cabo políticas comerciales activas.

En primer lugar, conviene no caer en los reduccionismos extremos que plantean que el Estado puede hacerlo todo, o bien que las fallas burocráticas son más importantes que las fallas de mercado y que por lo tanto no conviene hacer nada. La capacidad administrativa y la autonomía del Estado no son datos exógenos, sino que pueden ser modificados por las propias políticas públicas. En efecto, distintos estudios de organismos internacionales empiezan a refle- jar la necesidad de fortalecer (administrativa y presupuestariamente) a las instituciones públicas encargadas de la política comercial, industrial y tecnológica, para convertirlas en instrumentos, si no óptimos, por lo menos adecuados de intervención (OTA, 1990; Najmabadi, Banerji y Lall, 1992; CEPAL, 1990).

La existencia de casos exitosos de intervención sugiere que es posible aminorar considerablemente el peso de las fallas burocráticas. El fortalecimiento de las instituciones públicas no sólo contribuye a explicar los resultados tan diferentes que alcanzaron en el pasado los países asiáticos, por un lado, y los latinoamericanos, por otro, sino que también aparece como una condición y un desafío para el desempeño futuro de estos últimos.

En segundo lugar, los obstáculos para efectuar una intervención pública eficaz no son los mismos en todos los campos de acción. Es ampliamente aceptado que las políticas microeconómicas horizontales originan menos dificultades que las selectivas para obtener la información necesaria y generar oportunidades de captura de las oficinas públicas por intereses privados. A su vez, las polf́ticas específicas que se aplican en sectores con estructura de mercado más competitiva (fundamentalmente en aquellos con predominancia de empresas pequenas y medianas) parecen ser más fáciles de mantener bajo control que aquellas que se aplican a sectores altamente concentrados y con gran capacidad de cabildeo.

\section{VI}

\section{Conclusiones y recomendaciones de política}

En un panorama internacional de creciente globalización, y a la vez de profundización de las fricciones comerciales, los países de la región se ven en la necesidad de consolidar su inserción internacional, mejorando el perfil de sus exportaciones. El éxito de tal

\footnotetext{
29 Respecto a este tipo de argumentos véase Grossman (1986), Porter (1991) y los estudios sobre sociedades en busca de rentas econónicas, entre cuyos autores se destaca Krueger (1974).
}

empresa está indisolublemente ligado a procesos de reconversión que incrementen la eficiencia productiva y permitan incorporar nuevas ventajas comparativas. En ese contexto, la integración regional puede constituir un elemento catalizador que facilite y potencie los procesos de reconversión.

Esa reconversión deberá tener lugar, en algunos países, en medio de restricciones de política cambia- 
ria que eliminan toda receta fácil. Habrá que hacer hincapié en la aplicación de políticas que fomenten la otra vía fundamental para incrementar las exportaciones: el aumento de los niveles de productividad y de eficiencia comparativa. Hasta tanto estas medidas brinden sus frutos, la utilización de la política comercial como estrategia macroeconómica subóptima - para compensar posibles desviaciones en el tipo de cambio real, o como instrumento de apoyo en el frente fiscal- no debe ser descartada. Cabe aquí señalar, sin embargo, que cuando sea empleada con este propósito, debe respetarse la dimensión puramente macroeconómica de la política comercial. Esto supone no establecer diferencias sectoriales (que en todo caso deben fundamentarse en otro tipo de criterios) ni incrementar el sesgo antiexportador de la economía.

La estabilidad macroeconómica es una condición necesaria para el desarrollo de nuevas ventajas comparativas, pero no es por sí sola una garantía del mismo ${ }^{30}$ Una inserción internacional dinámica requiere además - como lo demostraron los países exitosos del Asia- una profundización de las políticas microeconómicas horizontales orientadas a aumentar la productividad y a consolidar posiciones en los mercados externos.

En el campo de la productividad es necesario definir una estrategia racional de políticas comerciales, industriales y tecnológicas optimas destinadas a atacar las importantes fallas de mercado que, en los países periféricos, afectan especialmente a los sectores tecnológicamente más avanzados: medidas de extensión industrial para la pequeña y mediana empresa (especialmente a fin de modernizar la organización del trabajo), promoción de actividades de investigación y desarrollo, incentivos a la formación de recursos humanos y otras.

Una de las áreas donde más queda por hacer es la de las políticas públicas dirigidas al desarrollo de la información sobre los mercados externos y a la promoción de empresas comercializadoras que hagan viables sobre todo las exportaciones de la pequeña y mediana empresa. Otro aspecto fundamental es la provisión de líneas de financiamiento para las exportaciones, a tasas de interés razonables. ${ }^{31}$

\footnotetext{
${ }^{30}$ Algunos autores llegan a asumir una posición muy crítica con lo que identifican como "el cortoplacismo de los esquemas de ajuste macroeconómico, que ignoran los horizontes más distantes que deberían orientar las decisiones estratégicas de los agentes públjcos y privados" (Tavares, 1990).

${ }^{31}$ Las subvenciones a la tasa de interés en los créditos a la exportación no han entrado en la lista de subsidios prohibidos por el GATT (Fundación U.I,A., Consejo Académico, 1994).
}

Otra instancia de política comercial que cabe destacar en este contexto es el avance de los procesos de integración regional. Estos procesos ofrecen la posibilidad de aumentar la productividad - aprovechando economías de escala y de especialización-, y de mejorar la capacidad negociadora en los foros internacionales. Sin embargo, para que los procesos de integración regional brinden todos sus frutos, deben lograr en su seno una amplia coordinación de políticas macroeconómicas y microeconómicas. La ausencia de tal coordinación y la persistencia de ciertas asimetrías a nivel microeconómico pueden hacer cristalizar en la región patrones de especialización intersectorial (del tipo Norte-Sur) que le impidan a algunos países aprovechar plenamente los beneficios de la integración.

Con respecto a la utilización de políticas comerciales selectivas (como la protección a ciertos sectores en reconversión), la experiencia de los países asiáticos nos muestra que ellas deben tener carácter transitorio y estar sujetas - como lo plantea el GATT para estos casos en el recientemente aprobado Código de Salvaguardias- al cumplimiento de ciertas metas por parte de los sectores protegidos. De allí que la aplicación de políticas comerciales selectivas en sectores altamente concentrados ( $y$ con capacidad de cabildeo) requiere como condición mínima que el Estado cuente con la capacidad institucional necesaria para fiscalizar adecuadamente el cumplimiento de los compromisos privados.

Esto plantea la imperiosa necesidad de llevar adelante una reconstrucción institucional que permita contar en el futuro con oficinas públicas más eficaces en la aplicación de instrumentos de política que, si bien son más complejos, pueden acercarse más al óptimo. Mientras tanto parece conveniente limitar en lo posible las políticas selectivas a aquéllas dirigidas a sectores con predominio de pequeñas y medianas empresas, donde la capacidad para disciplinar al sector privado puede ser mayor. ${ }^{32}$ Cabe destacar además que, como instrumentos de promoción selectiva, las barreras arancelarias o sistemas de cuotas licitadas por sobre arancel son superiores a las cuotas $u$ otras barreras no arancelarias, por su mayor transparencia (permiten definir el grado de protección que se le está brindando a un sector) y porque aumentan la recauda-

\footnotetext{
${ }^{32} \mathrm{Al}$ aplicar políticas comerciales selectivas debe tenerse en cuenta, además, la necesidad de evitar la generación de un sesgo antiexportador en el sector seleccionado y de una carga de protección efectiva negativa en aquellos sectores exportadores que utilizan los bienes protegidos.
} 
ción fiscal, reemplazando la generación de rentas casi nunca apropiadas por los consumidores.

Estas estrategias de política deben enmarcarse dentro de las nuevas pautas derivadas de la Ronda Uruguay. Como señalamos en la sección II, es muy probable que los acuerdos alcanzados en esa Ronda den lugar a diversas fricciones por las fuertes presiones proteccionistas en los países del Norte. Sin embargo, aún en este caso, la situación asimétrica que señalamos como una de las realidades del panorama intemacional puede obligar a los países periféricos a encuadrarse dentro de las nuevas normas. Partiendo de esta última hipótesis, cabe señalar aquí dos estrategias.

En primer lugar, en la medida en que las norma- tivas del GATT lleguen a limitar la utilización de ciertos instrumentos dirigidos a eliminar el sesgo antiexportador, habrá que usar otros instrumentos no prohibidos de incentivo a las exportaciones que contribuyan a aminorar dicho sesgo, como el otorgamiento de créditos a la exportación en condiciones favorables.

En segundo lugar, aun cuando las normativas del GATT impongan ciertas restricciones, hay plazos para ajustarse a ellas que es fundamental aprovechar (véase el anexo). Más aún, por la existencia de dichos plazos habrá que aprovechar al máximo, antes de su desmantelamiento, las políticas industriales y comerciales que se consideren adecuadas para consolidar el proceso de reconversión productiva.

\section{Bibliografia}

Amsden, A. (1986): The direction of trade past and present and the 'leaming effects' of export to different directions, Joumal of Development Economics, vol. 23, Amsterdam, Noth-Holland. (1989): Astats Next Giant. South Korea and Late Industrialization, Nueva York, Oxford University Press.

Bell, M., B. Ross-Larson y L. Westphal (1984): Assesing the Performance of Infant Industries, World Bank Staff Working Papers, $\mathrm{N}^{\circ}$ 666, Washington, D.C., Banco Mundial.

Brander, J. (1986): Rationales for strategic trade and industrial policy, P. Krugman (ed.), Strategic Trade Policy and the New International Economics, Cambridge, Mass., The MIT Press.

Brander, J. y B. Spencer (1981): Tariffs and the extraction of foreign monopoly rents under potential entry, Canadian Journal of Economics, $\mathrm{N}^{\circ} 14$, Toronto, Canadá, Canadian Economics Association.

CEPAL (Comisión Económica para América Latina y el Caribe) (1990): Transformación productiva con equidad. LC/G. 1601-P, Santiago de Chile, Publicación de las Naciones Unidas, $\mathbf{N}^{\circ}$ de venta S.90. II. G. 6.

Corden, M. (1974): Política comercial y bienestar económico, Madrid, ICE.

Dornbusch, R. (1990): Policy options for freer trade: The case for bilateralism, Lawrence y Schultze (eds.), An American Trade Strategy: Options for the 1990's, Washington, D.C., The Brookings Institution.

Dosi, G., C. Freeman, R. Nelson, G. Silverbeng y L. Soete (eds.) (1988): Technical Change and Economic Theory, Londres, Frances Pinter.

Dosi, G., K. Pavitt y L. Soete (1991): The Economics of Technical Change and International Trade, Londres, HarvesterWheatsheaf.

Fajnzylber, F. (1988): Competitividad internacional: evolución y lecciones, Revista de la CEPAL, No 36, LC/G. 1537-P, Santiago de Chite.

Fritsch, W. y G. Franco (1992): Politica comercial, de competiçấo e de investimento estrangeiro, Revista brasileira de comercio exterior, $\mathrm{N}^{\circ} 33$, Rio de Janejro, Fundación Centro de Estudios de Comercio Exterior (FUNCEX).

Fundación U.I.A. Consejo Académico (1994): La política comercial externa, Cuaderno $N^{\circ} 5$, Buenos Aires, Fundación Unión Industrial Argentina.

GATT (Acuerdo General sobre Aranceles Aduaneros y Comercio) (1993): Resumen: El acta final de la Ronda Uruguay, Focus. Boletin de Informacion, $\mathrm{N}^{\circ} 104$, Ginebra, diciembre.
Grimwade, N. (1989): Intemational Trade. New Patterns of Trade, Production and Investment, Londres, Routledge.

Grossman, G. (1986): Strategic export promotion: A critique, P. Krugman (ed.), Strategic Trade Policy and the New International Economics, Cambridge, Mass., The MIT Press.

Grossman, G. y E. Helpman (1991): Innovation and Growth in the Global Economy, Cambridge, Mass., The MIT Press.

Guerra Borges, A. (1993): Nuevo contexto mundial para America Latina, Integración latinoamericana, año $18, \mathrm{~N}^{\circ} 192$, Buenos Aires, Instituto para la Integración de América Latina (INTAL).

Guimarães, E. y L. Najdin (1994): GATT 1994: Os novos acordos sobre dumping, subsidios e salvaguardeas, Revista brasileira de comercio exterior, $\mathrm{N}^{\circ} 39$, Rio de Janeiro, FUNCEX.

Helpman, E. y P. Krugman (1985): Market Structure and Foreign Trade: Increasing Returns, Imperfect Competition and the International Economy, Cambridge, Mass., The MIT Press.

Hughes Hallets, A. J. (1994): The impact of the EC-92 on trade in developing countries, The World Bank Research Observer, Washington, D.C., Banco Mundjal, enero.

Hughes Hallets, A. J. y C.A Primo Braga (1994): The new regionalism and the threat of protectionism, mimeo.

Kaplinsky, R. (1988): Restructuring the capitalist labour process: Some lessons from the car industry, Cambridge Joumal of Economics, vol. 12, $\mathrm{N}^{\circ}$ 4, Londres, Academic Press Limited.

Krueger, A. (1974): The political economy of the rent-seeking society, The American Economic Review, vol. LXIV, No 3 , Los Angeles, California, American Economic Assocjation.

(1990): Free trade is the best policy, Lawrence y Schultze (eds.), An American Trade Strategy: Options for the 1990's. Washington, D.C., The Brookings Institution.

Krugman, P. (comp.) (1987): Strategic Trade Policy and the New International Trade, Cambridge, Mass. The MrT Press.

(1988): La nueva teoría del comercio internacional y los países menos desarrollados, El trimestre económico, vol. 55 , $\mathrm{N}^{\circ}$ 217, México, D.F., Fondo de Cultura Económica.

Laird, S. y J. Nogues (1989): Trade policies and the highly indebted countries, The World Bank Economic Review, vol. 3, $\mathrm{N}^{\circ} 2$, Washington, D.C., Banco Mundial.

Lawrence, R. (1993): Futures for the world trading system and their implications for developing countries, M. Agosin y D. Tussie (eds.), Trade and Growth. New Dilemmas in Trade Policy, Nueva York. St. Martin's Press.

Lucas, R. (1988): On the mechanics of economic development, Journal of Monetary Economics, vol. 22, Amsterdam, North-Holland. 
Martirena-Mantel, A.M. (1988): Distorsiones domésticas y ordenamiento óptimo de políticas comerciales en la economía abierta, Desarrollo económico, vol. 27, $\mathrm{N}^{\circ} 108$, Buenos Aires, Instituto de Desarrollo Económico y Social (IDES).

Motta Veiga، P. (1988): A inserção internacional da economia brasileira: condicionamentos e perspectivas, texto para discusión interna, $\mathrm{N}^{\circ} 15$, Rio de Janeiro, FUNCEX.

Najmabadi, F., S. Banerji y S. Lall (1992): World Bank Support for Industrialization in Korea, India, and Indonesia, Washington, D.C., Banco Mundial, Operations Evaluation Department.

Ocampo, J. A. (1993): New theories of international trade and trade policy in developing countries, M. Agosin y D. Tussie (eds.), Trade and Growth. New Dilemmas in Trade Policy. Nueva York, St. Martin's Press.

OTA (Oficina de Evaluación Tecnológica) (1990): Making Things Better. Competing in Manufacturing, Washington, D.C., Congreso de los Estados Unidos.

Porter, M. (1991): La ventaja competitiva de las naciones, Buenos Aires, Javier Vergara Editor $\mathbf{S . A .}$

Rhee, Y. (1989): Managing Entry into Intemational Markets: Lessons from the East Asian Experience, Industry series paper, $\mathrm{N}^{\circ}$ 11, Washington, D.C., The World Bank Industry and Energy Department.

Richardson, J.D. (1993): New Trade Theory and Policy a Decade
Old: Assessment in a Pacific Context, Reprint $\mathrm{N}^{\circ} 1814$, Cambridge, Mass., National Bureau of Economic Research.

Rodrick, D. (1993): Trade and Industrial Policy Reform in Developing Countries: A Review of Recent Theory and Evidence. Working paper, $\mathrm{N}^{\circ} 4417$, Cambridge, Mass., National Bureau of Economic Research.

Romer, P. (1986): Increasing returns and long-run growth, Joumal of Political Economy, vol. 94, No 5, Chicago, Ill., The University of Chicago.

Tavares, M.C. (1990): Reestructuración industrial y políticas de ajuste macroeconómico en los centros, R. Russell (ed.). El sistema internacional y América Latina. La agenda internacional en los años ' 90 . Buenos Aires, Programa de Estudios Conjuntos sobre las Relacjones Internacionales de América Latina (RIAL/Grupo Editor Latinoamericano).

Tyson, L. D. (1990): Managed trade: Making the best of the second best, Lawrence y Schultze (eds.), An American Trade Strategy: Options for the $1990^{\prime} \mathrm{s}$, Washington, D.C. The Brookings Institution.

Wade, R. (1990): Governings the Marker, Nueva Jersey, Princeton University Press.

Wonaccot. P. y R. Wonaccot (1981): Is unilateral tariff reduction preferable to a customs union? The curious case of the missing foreign tariffs, The American Economic Review, vol. $71, \mathrm{~N}^{\circ} 4$, Los Angeles, California, American Economic Association.

ANEXO

Nuevos instrumentos surgidos de la Ronda Uruguay del CATt

Entre los principales resultados de la Ronda Uruguay se encuentran tanto la creación de la Organización Mundial del Comercio (OMC), con un Consejo General encargado de supervisar el cumplimiento de los distintos acuerdos alcanzados y de resolver las disputas comerciales entre los países miembros, como la negociación de acuerdos sectoriales para la agricultura y el sector textil y la inclusión de los servicios y la propiedad intelectual en las negociaciones multilaterales.

Nos interesa aquí hacer referencia a los nuevos instrumentos diseñados para acentuar la disciplina en el comportamiento comercial. Estos incluyen nuevas normas para las inversiones relacionadas con el comercio; subsidios y derechos compensatorios; cláusulas de salvaguardia, y medidas antidumping.

\section{a) Medidas en materia de inversiones relacionadas con el comercio}

La existencia de medidas en materia de inversiones que pueden afectar al comercio llevó al GATT a prohibir una serie de prácticas en ese sentido. De especial relevancia es la prohibición de establecer normas sobre contenido nacional y medidas que tiendan a la nivelación del comercio (por ejemplo, relacionando la magnitud o el valor de las importaciones que puede realizar una empresa con el nivel de sus exportacio- nes). Se dio a los países en desarrollo un plazo de cinco años para eliminar este tipo de políticas.

\section{b) Subsidios y derechos compensatorios}

Se estableció una mayor disciplina en materia de subsidios, los que se clasificaron en tres categorías:

i) Subsidios prohibidos. Son aquellos que están vinculados al empleo de insumos internos o a metas de desempeño exportador. Sin embargo, para evitar la doble gravación, se acepta que continúen las exenciones a los impuestos indirectos sobre insumos incorporados en los productos exportados o que se apliquen mecanismos de reintegro. En estos casos los montos exceptuados o reintegrados no deben superar el valor de los impuestos efectivamente pagados.

ii) Subsidios no recurribles o permitidos. Estos se encuentran en el otro extremo. Incluyen los subsidios horizontales o no específicos, es decir, aquellos que no están limitados a determinados tipos de industria o actividad. Son subsidios no recurribles, por ejemplo, los orientados a aumentar el empleo, fomentar la pequer̃a y mediana empresa, u otorgar fondos para investigación y desarrollo. Se incluye también en esta categoría a los subsidios específicos, cuando se aplican para fomentar las actividades de investigación industrial, apoyar a regiones desfavorecidas, y llevar a cabo programas ambientales. Un país miembro pue- 
de, sin embargo, pedir la intervención del GATT si juzga que un subsidio que en principio es no recurrible está dañando seriamente una rama de su producción.

iii) Subsidios recurribles. En esta categoría cae el resto de los subsidios: son aquellos que pueden afectar adversamente los intereses de otros países y están sujetos a la demostración del daño sufrido. Se considera condición necesaria - aunque no suficiente- para que un subsidio sea recurrible que el total del subsidio ad valorem represente más del $5 \%$ del valor del producto. Los miembros afectados por subvenciones recurribles podrán someter la cuestion al órgano de solución de diferencias. Si se comprueban los efectos perjudiciales, el país que otorgó el subsidio deberá retirarlo o eliminar los efectos perjudiciales (GATT, 1993). Para los países en desarrollo con un ingreso per capita superior a los mil dólares la prohibición de subsidiar exportaciones entrará en vigor ocho años después de iniciarse la vigencia del acuerdo por el que se establece la OMC (GATT 1993).

El acuerdo estipula, a su vez, procedimientos más transparentes para la aplicación de derechos compensatorios sobre los productos importados que son subsidiados por terceros países. Las reglas para efectuar la apertura de las investigaciones son más estrictas, y se establece la necesidad de demostrar una relación causal directa entre tales importaciones y el daño efectivo que esté sufriendo la industria involucrada. Se pondrá fin a dichas investigaciones cuando la cuantía de la subvención sea muy pequeña (menos del $2 \%$ ad valorem) o el volumen real o potencial de las importaciones subsidiadas sea insignificante (menos del 4\% de las importaciones totales del producto similar que haga el importador).

\section{c) Acuerdo sobre Salvaguardias}

Este Acuerdo, incluido en el Acta Final de la Ronda Uruguay, introduce una modificación muy importante en el marco normativo destinado a defender segmentos industriales internos que hayan perdido competitividad. Para limitar comportamientos comerciales desleales que puedan encubrir acciones de proteccionismo arbitrario se establecieron reglas más claras para la aplicación del Código de Salvaguardias. Este puede utilizarse para proteger a la industria local cuando un aumento imprevisto de las importaciones amenace causar serios daños a los productores locales. En este caso, se autoriza a los gobiernos a suspender temporalmente los compromisos asumidos en el GATT y a establecer barreras al comercio por un plazo máximo de cuatro años (extensibles en ciertas circunstancias a hasta ocho años)..$^{33}$

Cuando el aumento de la protección responde a razones estrictas de menor eficiencia de la industria local, la aplicación de cláusulas de salvaguardia es claramente un instrumento más apropiado que las medidas antidumping o los derechos compensatorios. Estos últimos apuntan a penalizar a los productores externos sin exigir nada al productor local, mientras que la aplicación de salvaguardias presupone --al establecer un plazo para la protección- la exigencia de que el sector afectado lleve adelante un proceso de ajuste. En ese sentido, este último instrumento puede ser más beneficioso aún para el país que lo aplica (Guimarães y Naidin, 1994).

Por otro lado, se prohibe establecer acuerdos voluntarios de limitación de exportaciones o arreglos de comercialización ordenada entre las partes. Las medidas de este tipo existentes al firmarse el Acuerdo sobre Salvaguardias deberán ser eliminadas progresivamente en un plazo de cuatro años.

\section{d) El acuerdo antidumping}

Este acuerdo apunta a reglas más transparentes en la utilización de dicho mecanismo. Se intenta detallar y aclarar más el método para determinar la existencia de dumping, así como para calcular su magnitud. El país importador debe establecer en forma más estricta la relación causal entre las importaciones (sujetas a dumping) y el daño causado a la industria nacional. Se establecen además nuevas reglas para iniciar y conducir las acciones, y para posibilitar la defensa de las partes interesadas.

\footnotetext{
${ }^{33}$ Para facilitar el ajuste del sector afectado el Acuerdo sobre Salvaguardias establece que si el plazo previsto es superior a un año la medida de salvaguardia debe ser liberalizada progresivamente a intervalos regulares durante el petíodo de aplicación. Si la dura-
}

ción de la medida excede los tres aĩos la situación de la industria debe ser reanalizada periódicamente a fin de que la medida pueda ser levantada o el ritmo de liberalización acelerado. 